\title{
La supervision de mémoire en master : l'étudiant comme principal acteur de sa réussite
}

Laetitia Gerard

\section{(2) OpenEdition}

Édition électronique

URL : http://journals.openedition.org/ripes/407

ISSN : 2076-8427

Éditeur

Association internationale de pédagogie universitaire

Référence électronique

Laetitia Gerard, « La supervision de mémoire en master : l'étudiant comme principal acteur de sa réussite », Revue internationale de pédagogie de l'enseignement supérieur [En ligne], 26(2) | 2010, mis en ligne le 08 février 2011, consulté le 07 septembre 2020. URL : http://journals.openedition.org/ripes/ 407

Ce document a été généré automatiquement le 7 septembre 2020

Article L.111-1 du Code de la propriété intellectuelle. 


\title{
La supervision de mémoire en master : l'étudiant comme principal acteur de sa réussite
}

\author{
Laetitia Gerard
}

\section{Introduction}

1 En France, bon nombre d'étudiants semblent rencontrer des difficultés lors du passage de la licence ${ }^{1}$ au master ${ }^{2}$. Ces difficultés sont liées à l'introduction d'une nouvelle relation pédagogique, une relation duale qui prend la forme de la supervision de mémoire (Gérard, 2009). Pour essayer de comprendre les difficultés que rencontrent les étudiants dans le passage à une nouvelle relation pédagogique, nous avons mené des entretiens exploratoires auprès de douze étudiants inscrits en études de master. Les résultats ont révélé un lien entre la perception de l'étudiant quant à la qualité de sa supervision et sa réussite. L'étudiant en situation de réussite est considéré ici comme celui qui a obtenu une note à son mémoire de master qui lui permet d'accéder au niveau universitaire supérieur (Gérard, 2009). En effet, les étudiants qui se disaient satisfaits de leur direction avaient validé leur mémoire et obtenu une note satisfaisante. En revanche, contrairement à ce que nous pouvions penser, les résultats des étudiants peu ou pas satisfaits de leur direction étaient extrêmement hétérogènes : certains d'entre eux ont choisi d'abandonner leur mémoire en cours d'année, alors que d'autres l'ont mené à terme et validé avec succès. Nous sommes alors arrivés au questionnement suivant: pourquoi certains étudiants, qui jugent leur direction insatisfaisante, abandonnent-ils leur mémoire alors que d'autres parviennent à obtenir une note satisfaisante à leur travail de recherche? Qu'est-ce qui explique cette différence? Comment envisager la supervision de mémoire au niveau du master du point de vue de l'étudiant?

2 Dans cet article, nous ne nous intéressons pas à la qualité de la supervision et à ses effets sur la réussite de l'étudiant «masterant ». Nous nous intéressons plutôt au rôle 
de l'étudiant dans sa propre réussite, un aspect souvent négligé dans la littérature existante au sujet de la supervision (Delany, 2008 ; Leonard, Metcalfe, \& Evans, 2006). Nous entendons par «supervision » l'encadrement fourni par un enseignant-chercheur à un étudiant. Il s'agit d'une relation d'enseignement et d'apprentissage au sein de laquelle chaque acteur a un rôle à jouer. Cette relation peut s'inscrire à l'intérieur d'un programme de doctorat ou de master. Dans le cadre de cet article, nous nous concentrons sur la supervision en contexte de master. Nous verrons que la réussite en master passe par une forme d'autodirection de la part de l'étudiant et ce, quelle que soit la qualité de sa supervision. Les résultats de notre recherche montrent que cette posture d'autodirection de l'étudiant semble se traduire de deux manières différentes : d'une part, la découverte par l'étudiant des implicites de sa supervision et, d'autre part, l'utilisation de procédures de compensation pour ce qu'il peut considérer comme étant des « manques » dans sa supervision.

\section{Le rôle de l'étudiant dans sa réussite}

Une grande part des recherches qui portent sur la supervision de mémoires et thèses s'intéressent à la pratique de supervision, et plus précisément au lien entre la qualité de la supervision et la réussite de l'étudiant. À titre d'exemple, Zuber-Skerritt (1994) souligne qu'une bonne supervision de recherche est un moyen de réduire les abandons. Royer (1998) envisage également la supervision de recherche comme "un élément central des études doctorales » (Royer, 1998, p12) et Prégent (2001) l'analyse comme un important facteur d'abandon: « de trop nombreux candidats aux études supérieures ne terminent pas leur recherche à cause de difficultés reliées à l'encadrement » (Prégent, 2001, pxii). Plus récemment, Ives et Rowley (2005) mettent en évidence le lien entre la qualité de la supervision et la progression et la réussite de l'étudiant. Au vu des effets de la supervision sur la réussite aux deuxième et troisième cycles universitaires, des recherches ont émergé au sujet des bonnes pratiques de supervision dans le but d'améliorer la réussite des étudiants à ces niveaux universitaires (Delamont, Atkinson, \& Parry, 1997 ; Jutras, Ntebutse, \& Louis, 2010 ; Walker \& Thomson, 2010 ; Wisker, 2005).

Cependant, nous pouvons remarquer que les recherches qui portent sur les bonnes pratiques de supervision tendent à mettre de côté le rôle de l'étudiant dans sa propre réussite (Leonard, et al., 2006). Or, la relation pédagogique de supervision de recherche est une relation duale, le superviseur ne peut être le seul acteur de la réussite de l'étudiant. Dans la littérature, les quelques recherches qui s'intéressent à l'étudiant en situation de supervision de recherche, et non au superviseur, portent sur cinq principales thématiques :

1. Le processus de socialisation de l'étudiant "apprenti-chercheur» (Beauchamp, JazvacMartek, \& McAlpine, 2009 ; Green, 2005 ; Jazvac-Martek, 2009; Malfroy, 2005 McAlpine \& Norton, 2006 ; Shacham \& Od-Cohen, 2009);

2. La perception qu'il a de la qualité de sa supervision (Ives \& Rowley, 2005 ; Leduc, 1990 ; Powles, 1989);

3. Les compétences à développer à l'issu de son diplôme (Boud, 1991 ; Bruce \& Brameld, 1999 ; Gilbert, Balatti, Turner, \& Whitehouse, 2004 ; Kamler \& Thomson, 2004 ; Trafford \& Leshem, 2009) ;

4. Son rôle dans la relation pédagogique de supervision (Delamont, et al., 1997); 
5. La réussite de l'étudiant en fonction de ses caractéristiques personnelles, notamment la dimension genrée et la situation professionnelle de l'étudiant (Bowen \& Rudenstine, 1992 ; Conrad, 1994 ; Evans, 2006 ; Germeroth, 1991 ; Kember, 1999 ; Seagram, Gould, \& Pyke, 1998 ; Wright \& Cochrane, 2000).

5 Peu de recherches portent donc sur l'étudiant dans sa relation pédagogique de supervision et celles qui s'y intéressent ne portent pas sur le rôle de l'étudiant dans sa propre réussite. A notre connaissance, seule la recherche de Rudd (1985), l'une des recherches pionnières dans le champ de la supervision de recherche, fait mention de ce lien. Cette recherche anglosaxonne est ancienne, mais elle apporte quelques éléments de réflexion intéressants qui peuvent nous éclairer sur la situation française actuelle quant à la difficulté que les étudiants rencontrent dans leur passage de la licence en master et plus précisément dans leur passage d'une relation groupale d'enseignement à une relation duale de supervision.

6 Rudd (1985) a cherché à comprendre pourquoi certains étudiants confrontés à une mauvaise supervision ne réagissaient pas, c'est-à-dire qu'ils ne prenaient aucune initiative pour tenter d'améliorer leur situation. Il montre qu'il peut arriver que l'étudiant ne soit pas conscient des difficultés qu'il rencontre dans sa relation de supervision, notamment lorsqu'elles portent sur un mauvais choix de sujet, une absence d'aide à la planification ou sur le choix d'outils méthodologiques inadéquats. Il indique que, parfois, l'étudiant n'a personne à qui se plaindre de sa supervision, ou qu'il peut craindre que l'équipe de recherche et son superviseur de mémoire ne se retournent contre lui. Par ailleurs, le superviseur de recherche est, dans certains cas, le seul expert dans le domaine choisi par l'étudiant, ce qui écarte toute possibilité pour l'étudiant de changer de superviseur. Enfin, l'étudiant peut considérer son problème de supervision comme un problème privé qui, à ce titre, doit rester au sein même du binôme. À cet effet, une exposition publique risquerait d'aggraver la situation en suscitant la rancœur du superviseur.

Ceci nous porte à croire que, face à une mauvaise supervision, l'étudiant développe des compétences d'autodirection dans la gestion de la relation pédagogique duale, notamment dès qu'il parle de sa mauvaise supervision à quelqu'un. Nous nous appuyons ici sur la définition de l'apprentissage autodirigé de Knowles (1975). Ce dernier envisage l'apprentissage autodirigé comme: «...un processus dans lequel les individus prennent l'initiative, avec ou sans l'aide des autres, pour faire le diagnostic de leurs besoins et formuler leurs objectifs d'apprentissage, pour identifier les ressources humaines et matérielles pour apprendre, pour choisir et mettre en oeuvre les stratégies d'apprentissage appropriées et pour évaluer les résultats des apprentissages réalisés. » (Knowles, 1975, p. 77). Ainsi, nous pouvons formuler l'hypothèse que la réussite de l'étudiant passe par une forme d'autodirection de sa part. Nous avons donc cherché à déterminer quelle(s) forme(s) prend l'autodirection chez les étudiants de master en situation de réussite, particulièrement lorsqu'ils jugent leur supervision inadéquate.

\section{Méthodologie}

8 L'objectif de la recherche était, premièrement, de déterminer si l'étudiant faisait preuve d'autodirection dans sa supervision de mémoire ou s'il tendait plutôt à rester dans une posture inactive comme celle décrite par Rudd (1985). Deuxièmement, l'objectif était de décrire quelle(s) forme(s) prenait cette autodirection. Pour cela, nous 
avons choisi d'interroger vingt binômes «superviseur/étudiant » de manière à pouvoir confronter leurs interprétations et leurs expériences liées à la supervision vécue. Plus précisément, nous cherchions à obtenir des informations sur leur perception du comportement d'autodirection de l'étudiant. Le superviseur et l'étudiant ont été interviewés une première fois en milieu d'année et une seconde fois après la soutenance de l'étudiant (ou en fin d'année en cas d'abandon). L'objectif du second entretien était de :

- Recueillir des données sur le déroulement de la soutenance de l'étudiant ;

- Recueillir des informations sur la note obtenue au mémoire et à la présentation orale du mémoire (soutenance orale), en prenant aussi en considération l'appréciation de l'étudiant sur son travail de recherche et sur sa notation, ainsi que l'appréciation de son superviseur sur ces deux aspects (qualité du mémoire et notation). Ces données visaient à " évaluer » la réussite de l'étudiant ;

- Recueillir des données sur l'évolution de la relation pédagogique entre le superviseur et son étudiant à la fin du diplôme.

Les vingt binômes appartiennent à trois disciplines des sciences humaines et sociales (sciences de l'éducation, psychologie et sociologie). Le choix s'est fixé sur des étudiants en deuxième année de master, et non en première année, parce que les étudiants de master 2 ont déjà fait l'expérience de la supervision pendant les études de master 1 . Ils possèdent donc une distance critique et une expérience de la supervision qui en favorisent leur analyse. Nous avons pris contact avec les étudiants et les superviseurs par courrier électronique. Ce courriel présentait les objectifs de la recherche et sollicitait un entretien sur la base du volontariat. Dans les sept laboratoires de recherche qui ont accepté de participer à l'enquête (sur 24 contactés), cinquante-six superviseurs de mémoire et cent cinquante-six étudiants ont été sollicités. Au total, nous avons eu 27 accords d'entretien de la part des étudiants et 26 de la part des superviseurs. Nous avons choisi d'interroger l'ensemble de ce groupe d'étude, qui se répartissait sur sept sites universitaires français. Parmi ce groupe d'étude, nous avons vingt binômes "superviseur/étudiant» (cf. tableau 1). À noter que deux étudiants bénéficient d'une co-direction assurée par deux superviseurs. Typiquement, l'un des superviseurs est généralement un professeur qui possède l'habilitation à diriger des recherches (HDR) et l'autre est un maître de conférences qui, lui, ne possède pas l'HDR.

Tableau 1. Catégorisation des binômes en fonction du genre du superviseur, du genre de l'étudiant et de l'origine de la formation dont est issu l'étudiant.

\begin{tabular}{|c|c|c|c|c|}
\cline { 2 - 5 } \multicolumn{1}{c|}{} & Type de formation & Superviseur & Superviseure & Total \\
\hline \multirow{2}{*}{ Étudiante } & $\begin{array}{c}\text { Formation continue } \\
\text { (étudiants en reprise d'études) }\end{array}$ & 2 & 5 & 7 \\
\cline { 2 - 5 } & Formation initiale & 4 & 2 & 6 \\
\hline \multirow{2}{*}{ Étudiant } & Formation continue & 4 & 1 & 5 \\
\cline { 2 - 5 } & Formation initiale & 3 & 1 & 4 \\
\hline \multicolumn{2}{|c}{ Total } & 13 & 9 & 22 \\
\hline
\end{tabular}

Le superviseur et son étudiant ont été interrogés séparément par entretien semidirectif, de manière à ce qu'ils puissent s'exprimer plus librement et parler l'un de l'autre sans retenue. Nous avons élaboré le guide d'entretien à partir de vingt entretiens exploratoires (Quivy \& Van Camphenhoudt, 2006) menés en amont de cette étude auprès de six superviseurs et six étudiants de master. Les résultats de ces 
entretiens exploratoires nous ont permis, d'une part, de dégager une certaine exhaustivité dans les thèmes abordés. D'autre part, ils ont servi au pilotage du protocole d'entretien.

11 Le guide d'entretien s'organise autour de cinq grandes thématiques: 1) le premier contact entre le superviseur et l'étudiant ; 2) le déroulement des rencontres ; 3) l'aide apportée par le superviseur dans la construction du travail de recherche de l'étudiant ; 4) la relation inter-personnelle au sein du binôme ; 5) la perception du rôle de chacun et leurs attentes.

Les entretiens ont duré entre 30 minutes et $1 \mathrm{~h} 30$. Ils ont été enregistrés, retranscrits dans leur intégralité et anonymisés. Nous avons analysé les transcriptions d'entretiens par codage émergeant (Mucchielli, 1993) à l'aide du logiciel de traitement de données qualitatives NVivo (Welsh, 2002). Nous avons ensuite découpé le corpus en unités de sens, regardé le contenu pour le "ranger » dans la ou les boîte(s) thématique(s) appropriée(s). La segmentation s'est réalisée par paragraphe de manière à ne pas trop décontextualiser le discours.

13 Illustrons la technique. L'extrait suivant a été "rangé » dans la boite thématique "premier contact entre le superviseur et l'étudiant», car il y est question de la constitution des binômes.

Interviewer : et le choix du directeur comment il se fait?

D2 : euh/c'est vraiment les étudiants qui choisissent hein/alors il y a une pratique mais moi je ne cautionne pas/en fait traditionnellement on a une réunion fin décembre environ/où les étudiants parlent de tout ce qu'ils ont envie de faire/et c'est là en général que le choix du directeur se fait/c'est souvent l'étudiant qui euh/en fonction des domaines de recherche des chercheurs des enseignants il va s'orienter vers tel ou tel directeur de mémoire/ou alors ça peut arriver qu'on suive des étudiants depuis la maîtrise depuis le M1/et où le mémoire de M2 peut être un prolongement du mémoire de M1/d'ailleurs maintenant c'est plutôt ce qu'on va essayer de/de faire/

Nous avons procédé ensuite à une analyse thématique intrabinôme, c'est-à-dire à une comparaison des discours au sein des binômes. Par exemple, concernant l'un des sousthèmes qui porte sur l'évaluation du mémoire, en confrontant le discours du superviseur D12 avec celui de son étudiant E11, nous pouvons remarquer qu'il y a une différence entre la manière dont le superviseur décrit l'écrit obligatoire à remettre par l'étudiant en cours d'année et la description qu'en fait son étudiant :

"On a demandé [aux M2], à mi-étape, de produire un document de cinq pages dans lequel on leur demande de faire état de leur travail » (D12).

"Il y avait aussi un travail qu'il fallait rendre [...] on a un examen ici on a un premier examen où vous présentez, tu fais, disons, la problématique, les hypothèses les objectifs, en cinq pages [...] tu fais ta présentation les profs te posent des questions et tu es noté par rapport à ça » (E11).

E11 conçoit cet écrit comme un exercice à réaliser, qui doit répondre au mieux aux attentes du "prof ", comme il le nomme, de manière à obtenir une note satisfaisante. Cette attitude que nous qualifierions d' "attitude scolaire » vis-à-vis de l'écrit imposé montre la difficulté de l'étudiant à faire son passage. Il se considère encore comme un étudiant qui doit rendre un devoir en fin d'année, alors que son superviseur attend plutôt qu'il se place dans une posture d'apprenti-chercheur qui se forme à la recherche par la recherche et qui produit diverses ébauches dans le cadre de la réalisation d'une recherche. 


\section{Le lien entre autodirection et réussite en master}

Notre deuxième entretien a permis de mettre en exergue cinq situations de supervision :

1. Treize étudiants sont en situation de réussite : ils ont validé leur mémoire en une année et ils ont obtenu une note qui leur permet de poursuivre en doctorat ;

2. Deux étudiants sont en situation correspondant à ce que nous désignons comme une réussite "de la seconde chance ": ils ont dû soutenir leur mémoire deux fois (à la première et à la deuxième session) ou se réinscrire une année supplémentaire pour valider leur mémoire et obtenir une note qui leur permette de poursuivre au doctorat ;

3. Un étudiant est en situation d'échec partiel : il a validé son mémoire mais la note obtenue ne lui permet pas de poursuivre au doctorat ;

4. Un étudiant est en situation d'échec : il a soutenu son mémoire, mais ne l'a pas validé, c'està-dire qu'il a obtenu une note inférieure à dix sur vingt ;

5. Trois étudiants ont abandonné le master.

A partir de l'analyse intrabinôme, nous pouvons remarquer que les étudiants en situation de réussite (réussite et réussite " de la seconde chance ») sont ceux qui font le plus preuve d'autodirection. A titre d'exemple, l'étudiante E3, en situation de réussite, et l'étudiante E21 en situation de réussite de la « seconde chance » préparent une liste de questions pour chacun de leurs rendez-vous :

"[Je prépare] toujours une liste de questions et je note au fur et à mesure quand ça me vient,

je ne viens pas sans rien " (E3)

"J'ai ma petite liste de questions tout le temps pour ne rien oublier et je pose des questions

au fur et à mesure on y répond au fur et à mesure » (E21).

La posture d'autodirection semble se traduire de deux manières différentes : la capacité de l'étudiant à découvrir les éléments implicites de sa supervision et son utilisation de procédures de compensation pour ce qu'il pourrait considérer comme étant des «manques» dans sa supervision.

\subsection{La découverte des implicites de la supervision}

D'après nos données, nous pouvons remarquer que les étudiants en situation de réussite sont ceux qui parviennent à découvrir les implicites de leur supervision et à se les approprier. L'extrait suivant décrit la nature des implicites que l'étudiant E4, en situation de réussite, est parvenu à découvrir et à s'approprier :

"Ça c'est implicite mais quand un directeur de recherche accepte de t'accompagner, eh bien il attend de toi des résultats en fait, il attend que tu avances [...] il attend que tu découvres, il attend que dans ton mémoire des choses soient intéressantes " (E4, étudiant en situation de réussite).

"Si on attend dans son coin que c'est le directeur qui vienne dire, mais où est-ce que tu en es, moi je trouve que c'est plutôt insultant, moi je crois qu'il ne faut pas arriver à ce stade-là » (E4).

A l'inverse, les étudiants en situation d'échec tendent à ne pas percevoir les éléments implicites de leur rôle. On observe par exemple un hiatus entre l'étudiant E12 et sa superviseure quant à leur conception du rôle d'un superviseur relatif à l'aide bibliographique à apporter à l'étudiant. E12 attend un apport bibliographique principal (Gérard, 2009), c'est-à-dire une aide bibliographique apportée par son superviseure en amont de son travail de recherche bibliographique, et qui se traduit par la mise à 
disposition de l'étudiant d'une première bibliographie des principaux ouvrages à lire pour sa recherche :

"Tout le cadre théorique tout le cadre de connaissances n'était pas présent et on ne m'a pas non plus dit bon il faudrait que tu lises ce bouquin-là, ça a été très autonome " (E12, étudiant en situation d'échec).

21 Sa superviseure envisage, quant à elle, que son rôle consiste à fournir une aide bibliographique complémentaire (Gérard, 2009) à l'étudiant, c'est-à-dire à fournir à l'étudiant quelques références bibliographiques qui viennent compléter sa recherche bibliographique préalable. Elle ajoute les références bibliographiques en aval, après un travail personnel de l'étudiant :

"Par exemple là je viens de renvoyer des idées lumineuses de bibliographie à un étudiant que je suis [...] depuis deux ans parce qu'au hasard moi je tombe sur des trucs qui peuvent l'intéresser 》 (D17).

On remarque par ailleurs que cet étudiant en situation d'échec tend à s'interroger sur son rôle uniquement lorsqu'il rencontre une difficulté :

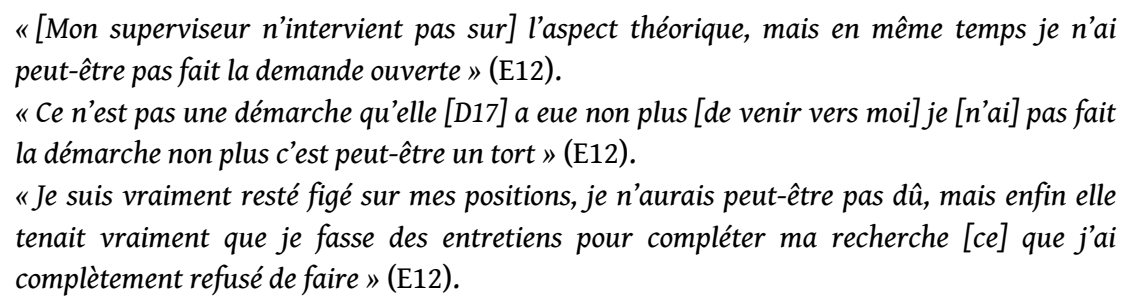

\subsection{L'utilisation de procédures de compensation}

Deuxièmement, on observe chez les étudiants en situation de réussite des comportements compensatoires. Par exemple, l'étudiante E3 exprime ses difficultés d'utilisation d'un nouvel outil méthodologique pour lequel ni elle ni sa superviseure n'étaient compétentes «je ne savais pas exactement ce que je devais filmer 》 (E3, étudiante en situation de réussite). Elle a su adopter un comportement compensatoire en sollicitant l'aide d'un pair :

"[J'ai] demandé [à une étudiante doctorante] de me passer son mémoire de master 2, je savais qu'elle avait utilisé la vidéo » (E3).

Pour prendre un autre exemple, on trouve également des procédures compensatoires dans le discours de l'étudiante E14. Cette étudiante n'a pas obtenu l'aide méthodologique escomptée de la part de son superviseur et ce, bien qu'elle lui ait explicité cette attente d'aide. Elle a donc choisi de compenser ce manque par la sollicitation d'une aide extérieure auprès d'un pair, en l'occurrence une doctorante, dont elle jugeait acquise la compétence de recherche :

"J'ai une de mes meilleures amies qui est en thèse [...] voyant que je pédalais dans la semoule elle m'a dit je vais te filer un coup de main [...] alors c'était échange de bon procédé moi je la relisais, j'ai une formation littéraire, moi j'avais besoin d'un coup de main méthodologique » (E14, étudiante en situation de réussite).

On observe d'ailleurs une différence dans les procédures de compensation utilisées par les étudiants en formation continue (FC) versus ceux en formation initiale (FI). Les étudiants en formation continue tendent à compenser davantage leur manque que les étudiants en formation initiale et ils ont tendance à compenser par la sollicitation de personnes ressources (les pairs, des collègues de travail ou des enseignants). 
"Il y avait une nouvelle technologie à apprendre, c'est-à-dire comme je suis allé avec des caméras, des petits enregistreurs et tout donc j'ai proposé de faire un petit stage là, il a directement envoyé mon souhait à l'espace recherche qui m'ont dit mais monsieur il n'y a pas de problème " (E4, étudiant en FC en situation de réussite).

"Il n'avait pas beaucoup de connaissances méthodologiques sur comment filmer sur le terrain et ça il l'a appris, il l'a relativement maîtrisé pour un M2, tout en faisant un travail solide» (D7, superviseur de E4).

Les étudiants en formation initiale montrent peu de procédures de compensation pour résoudre leurs difficultés qu'ils considèrent comme des manques dans leur supervision. Beaucoup d'étudiants en formation initiale ne compensent d'ailleurs aucun de leurs manques et tendent à rester avec leurs insatisfactions. Nous pourrions parler alors de procédures compensatoires négatives, qui permettent à l'étudiant de diminuer son sentiment d'insatisfaction par rapport à ces manques, mais ne comblent pas ses manques en tant que tels, et se réalisent souvent au détriment de la formation à la recherche par la recherche :

"Quand je voyais toutes ses corrections, je me disais, mais je n'arriverai jamais à terminer mon mémoire enfin, un truc pareil autant tout refaire direct, au départ je m'étais un peu découragée les quinze premiers jours, après je me suis dit ne t'inquiète pas tu fais ton truc toute seule tu ne l'écoutes plus et tout va très bien, j'étais épanouie personnellement et tout se passait bien " (E26).

Par ailleurs, on constate que pour les étudiants en formation initiale qui compensent leurs manques, les procédures de compensation prennent souvent la forme de lecture d'ouvrages méthodologiques ou le suivi des cours méthodologiques de M1. Cela ne prend jamais la forme d'une sollicitation d'une aide à une personne extérieure. Les étudiants en formation initiale tendent alors à compenser avec les outils estudiantins qu'ils connaissent, tels que l'utilisation de ressources documentaires, les stratégies estudiantines (par exemple, se servir des échéances des séminaires pour compenser ses difficultés à prendre en charge la planification) ou le suivi des cours de M1, c'est ce que nous nommons la compensation académique. Ainsi, ils utilisent des outils de compensation qu'ils ont fort probablement utilisés au cours des années antérieures en licence et qui ont fonctionné. La compensation académique privilégie une visée de réussite au diplôme, en permettant à ces étudiants de coller au mieux aux attentes de leur superviseur, l'approche «stratégique » à l'égard des études envisagée par Ramsden (2003), mais sans nécessairement favoriser le développement des compétences de recherche :

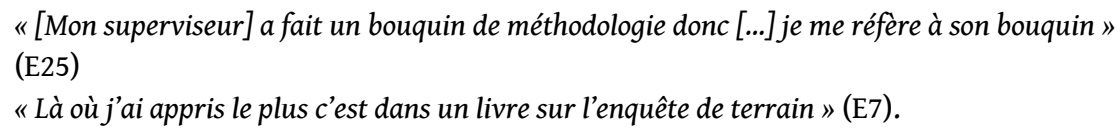

$28 \mathrm{Au}$ contraire, les étudiants en formation continue sont plutôt dans ce que nous nommons l'éco-compensation basée sur la notion d' "éco-formation " développée par Pineau et Michele (1983), c'est-à-dire la compensation par la sollicitation d'une aide extérieure trouvée dans leur environnement. Nous définissons ici l'éco-compensation comme une compensation qui s'effectue par appui sur d'autres personnes que son superviseur.

29 Là encore, on remarque que les étudiants en situation d'échec ou d'abandon sont plutôt restés dans l'inactivité décrite par Rudd (1985), ils ne compensent pas leurs «manques» et restent avec leur insatisfactions. Par exemple, le superviseur de E7 considère que :

«Le travail [...] en master 2 [...] exige [...] une certaine autonomie» (D9). 
individuels :

"Ils savent que c'est à eux de me solliciter » (D9).

31 sollicitation :

"[Ce que] j'attendrais de lui ce serait de prendre plus de mes nouvelles, en me disant ben là

tu en es où, tu as besoin d'aide » (E7, étudiante en situation d'abandon).

Le passage à l'autodirection n'a pas eu lieu. Ses difficultés à s'autodiriger ont eu un effet négatif sur son passage au statut d'apprentie-chercheure. Par exemple, elle n'a rendu aucun écrit de sa propre initiative à son superviseur, le passage à l'écriture scientifique n'a donc pas eu lieu non plus.

\section{Encourager l'autoréflexion pour développer l'autodirection}

Nous venons de relever que les étudiants en situation de réussite sont ceux qui, d'une part, tendent à découvrir les implicites de leur supervision et à se les approprier et ceux qui, d'autre part, adoptent des procédures de compensation pour combler ce qu'ils pourraient considérer comme étant des manques dans leur supervision. Ces deux comportements d'autodirection chez l'étudiant, qui semblent favoriser sa réussite, pourraient être encouragés par une activité réflexive sur sa relation de supervision.

Kolb (1984) considère le processus réflexif comme un élément essentiel de l'apprentissage expérientiel. Pearson et Brew (2002) parlent de l'apprentissage expérientiel pour décrire la professionnalisation à la pratique de supervision de recherche. Ils considèrent que l'approche la plus efficace en termes de formation à cette pratique pour l'apprenant est la mise en mots de sa pratique : ce qu'il fait et pourquoi il le fait. La mise en mots pourrait donc avoir un effet bénéfique chez l'étudiant dans sa compréhension, puis dans la gestion de sa relation pédagogique de supervision. En effet, c'est par la réflexion sur son expérience que l'étudiant parviendrait à la fois à découvrir les implicites et trouver des stratégies de compensation bénéfiques. Le modèle d'apprentissage de Kolb (1984) décrit un cycle expérientiel en quatre phases qui alterne action et réflexion: l'expérience concrète, l' observation réflexive, la conceptualisation abstraite et l'expérimentation active. L'individu entre par l'expérience concrète, il identifie une problématique ou un questionnement qu'il a rencontré. Il analyse cette expérience, c'est l'observation réflexive : il identifie les points qui posent question. Ensuite, il va en faire une généralisation, une théorie personnelle, applicable à la plupart des situations identiques. Nous sommes ici dans la conceptualisation abstraite. Enfin, cette généralisation va être transférée dans la pratique, c'est l'expérimentation active, pour résoudre la problématique ou les questionnements initiaux.

Dans le cas qui nous intéresse, l'étudiant entre dans l'expérience concrète lorsqu'il reçoit son premier écrit annoté par son superviseur. Il examine les feedbacks reçus puis entre dans une phase d'observation réflexive: il constate, par exemple, qu'il y a énormément d'annotations sur son texte et que les feed-backs ne portent que sur le fond et non sur la forme (syntaxe, grammaire et orthographe). Il en tire des conclusions et entre dans la phase de conceptualisation abstraite. A la lecture des annotations, il s'aperçoit que les 
feedbacks sont constructifs et que son superviseur ne corrige pas les fautes de syntaxe, grammaire et d'orthographe. Il peut, dès lors, envisager un scénario, il s'agit de l' expérimentation active, puisqu'il a découvert une clause implicite qui consiste à rendre des écrits régulièrement à son superviseur pour progresser dans sa recherche. La procédure de compensation consistera à faire relire ses écrits par un pair pour corriger les erreurs de syntaxe, grammaire et d'orthographe.

Dans le champ de l'autoformation, il existe des outils qui peuvent favoriser l'autoréflexion de l'étudiant. Clénet (2006), par exemple, propose le journal de bord ou les récits d'expérience, pour contribuer au développement de l'autoréflexion de manière à "favoriser le questionnement de son expérience " pour encourager "l'analyse critique " via "l'exploration, l'explicitation et la prise de conscience " (Clénet, 2006, p. 119). Barbot (2006) propose, quant à elle, les «journaux d'étonnement ». Le but est également de développer l'autoréflexion de l'apprenant à partir et sur son expérience. Son outil a été initialement proposé à des étudiants français stagiaires à l'étranger (futurs enseignants du français à l'étranger). Ils étaient invités à rédiger ce que l'auteure nomme un "journal d'étonnement» dans le but d'apprendre à mieux vivre la différence culturelle entre leur pays d'origine, la France, et le pays d'accueil, le Royaume-Uni. La consigne était de «noter tous les étonnements positifs heureux, mais aussi négatifs, d'en chercher la cause et de la noter » (Barbot, 2006, p. 184). La démarche comprend un objectif psychologique «destiné à aider les stagiaires placés dans une école anglaise à noter leurs coups de cœur et leurs rejets, à chercher à les analyser : cet outil devait faciliter la prise de conscience du processus que les stagiaires vivaient» (Barbot, 2006, p. 184). Mais elle est aussi «déclencheur d'interactions" selon les termes de l'auteure, dans le sens où Barbot proposait une synthèse des "journaux d'étonnement", ouverte à la discussion. Les journaux d'étonnement possèdent donc à la fois une dimension individuelle, la mise en mots par l'écriture permettant une autoréflexion du stagiaire, et une dimension collective, qui favorise l'autoréflexion par l'interaction entre pairs.

\section{Conclusion}

Les résultats de cette recherche nous amènent à nous questionner sur la réussite en master, non pas en nous interrogeant sur les bonnes pratiques de supervision, mais en nous interrogeant sur la manière de favoriser l'autoréflexion de l'étudiant. En prenant appui sur les travaux de Clénet (2006) et Barbot (2006), nous recommandons l'usage d'une démarche réflexive qui favorise le processus d'autoréflexion de l'étudiant. Nous conseillons au superviseur de mémoire de suggérer à l'étudiant de tenir un « journal de bord » de manière à favoriser, par la mise en mots et par la prise de distance, son autoréflexivité et une posture d'autodirection favorable à la réussite. Néanmoins, nous recommandons plutôt le recours à un outil «verbal " plutôt qu'à un outil « scriptural » tel le journal de bord, par exemple, en organisant des «tribunes libres». Celles-ci peuvent être animées une à trois fois dans l'année par une personne " neutre ", c'est-àdire une personne qui n'appartient pas au laboratoire de recherche. Les étudiants sont alors invités à s'exprimer librement sur la manière dont ils perçoivent leur supervision et à nommer les difficultés qu'ils rencontrent dans cette relation.

Nous nous sommes intéressés dans cette étude au rôle de l'étudiant dans sa réussite. Nous avons constaté que l'étudiant est le principal acteur de sa réussite notamment de 
par sa capacité d'autodirection. Nos données montrent que l'autodirection des étudiants en situation de réussite se traduit de deux manières avec, d'une part, la découverte des implicites et, d'autre part, la capacité de compensation des «manques». Il y a néanmoins plusieurs limites à notre recherche exploratoire. Tout d'abord, il s'agit d'un petit groupe d'études comprenant vingt binômes et d'une enquête réalisée dans le cadre de seulement trois disciplines des sciences humaines et sociales. Il faudrait élargir le groupe d'études à d'autres disciplines des sciences humaines et sociales, mais aussi aux disciplines scientifiques. Finalement, il convient de relever qu'il s'agit d'une recherche française et les particularités du système universitaire français ne permettent pas toujours de transférer les résultats obtenus à d'autres contextes.

\section{BIBLIOGRAPHIE}

Barbot, M. J. (2006). L'accompagnement de l'expérience interculturelle : Construire la rencontre ? In H. Bezille \& B. Courtois (Eds.), Penser la relation experience-formation (pp. 171-187). Lyon : Chronique Sociale.

Beauchamp, C., Jazvac-Martek, M., \& McAlpine, L. (2009). Studying doctoral education : Using Activity Theory to shape methodological tools. Innovations in Education and Teaching International, 46(3), 265-277.

Boud, D. (1991). Implementing student self-assessment. Campbelltown : HERDSA.

Bowen, W. G., \& Rudenstine, L. N. (1992). In Pursuit of the PhD. New Jersey : Princeton University Press.

Bruce, C. S., \& Brameld, G. H. (1999). Encouraging student-directed research and critical thinking. In Y. Ryan \& O. Zuber-Skerritt (Eds.), Supervising postgraduate students from non-english speaking backgrounds (pp. 157-166). Buckingham, UK : The society for research into higher education \& Open university press.

Clénet, C. (2006). L'accompagnement de l'autoformation expérientielle. In H. Bezille \& B. Courtois (Eds.), Penser la relation experience-formation (pp. 113-127). Lyon : Chronique Sociale.

Conrad, L. (1994). Gender and postgraduate supervision. In O. Zuber-Skerritt \& Y. Ryan (Eds.), Quality in postgraduate education (pp. 51-58). London : Kogan Page.

Delamont, S., Atkinson, P., \& Parry, O. (1997). Supervising the PhD : A guide to success. Buckingham : Open university press.

Delany, D. (2008). A review of the literature on effective PhD supervision. Trinity College : Centre for Academic Pratice and Student Learning (CAPSL).

Evans, T. (2006). Part-time candidature - balancing candidature, work and personal life. In C. Denholm \& T. Evans (Eds.), Doctorate downunder : Keys to successful doctoral study in Australia and New Zealand (pp. 137-144). Camberwell : ACER Press. 
Gérard, L. (2009). L'accompagnement en contexte de formation universitaire : Etude de la direction de mémoire comme facteur de réussite en Master. Unpublished Thèse de doctorat, Université Nancy 2 , Nancy.

Germeroth, D. (1991). Lonely days and lonely nights : Completing the doctoral dissertation. Bulletin of the association of canadian archivists, 76, 60-89.

Gilbert, R., Balatti, J., Turner, P., \& Whitehouse, H. (2004). The generic skills debate in research higher degrees. Higher education research and development, 23(3), 375-388.

Green, B. (2005). Unfinished business : Subjectivity and supervision. Higher education research and development, 24(2), 151-163.

Ives, G., \& Rowley, G. (2005). Supervisor selection or allocation and continuity of supervision : PhD students' progress and outcomes. Studies in higher education, 30(5), 535-555.

Jazvac-Martek, M. (2009). Oscillating role identities : The academic experiences of education doctoral students. Innovations in education \& teaching international, 46(3), 253-264.

Jutras, F., Ntebutse, J. G., \& Louis, R. (2010). L'encadrement de mémoires et de thèses en sciences de l'éducation : Enjeux et défis. Revue internationale de pédagogie de l'enseignement supérieur, 26(1).

Kamler, B., \& Thomson, P. (2004). Driven to abstraction : doctoral supervision and writing pedagogies. Teaching in Higher Education, 9(2), 195-209.

Kember, D. (1999). Integrating part-time study with family, work and social obligations. Studies in higher education, 24(1), 109-124.

Knowles, M. (1975). Self-directed learning. Chicago : Follet.

Kolb, D. A. (1984). Experiential learning : Experience as the source of learning and development. Englewood Cliffs : Prentice-Hall.

Leduc, A. (1990). La direction des mémoires et des thèses. Brossard : Behaviora

Leonard, D., Metcalfe, J., \& Evans, J. (2006). Review of literature on the impact of working context and support on the postgraduate research student learning experience. London : The Higher Education Academy.

Malfroy, J. (2005). Doctoral supervision, workplace research and changing pedagogic practices. Higher Education Research \& Development, 24(2), 165-178.

McAlpine, L., \& Norton, J. (2006). Reframing our approach to doctoral programs : An integrative framework for action and research. Higher education research and development, 25(1), 3-17.

Mucchielli, R. (1993). Le questionnaire dans l'enquête psycho-sociale. Connaissance du problème, aplications pratiques. Paris : ESF.

Pearson, M., \& Brew, A. (2002). Research training and supervision development. Studies in Higher education, 27(1), 135-150.

Pineau, G., \& Michèle, M. (1983). Produire sa vie : autoformation et autobiographie. Paris : Edilig.

Powles, M. (1989). How's the thesis going ? Former postgraduates' and their supervisors' views on lengthy candidature and dropout. Melbourne : University of Melbourne.

Prégent, R. (2001). L'encadrement des travaux de mémoire et de thèse. Montréal : Presses internationales polytechnique.

Quivy, R., \& Van Camphenhoudt, L. (2006). Manuel de recherche en sciences sociales. Paris. 
Ramsden, P. (2003). Learning to teach in higher education (2nd edition ed.). New York : RoutledgeFalmer.

Royer, C. (1998). Vers un modèle de direction de recherche doctorale en sciences humaines. Sainte-Foy : Presses de l'Université du Québec.

Rudd, E. (1985). A new look at post graduate failure. Guilford : SRHE \& NFER-Nelson.

Seagram, B. C., Gould, J., \& Pyke, S. W. (1998). An investigation of gender and other variables on time to completion of doctoral degrees. Research in higher education, 39(3), 319-335.

Shacham, M., \& Od-Cohen, Y. (2009). Rethinking PhD learning incorporating communities of practice. Innovations in Education and Teaching International, 46(3), 279-292.

Trafford, V., \& Leshem, S. (2009). Doctorateness as a threshold concept. Innovations in education and teaching international, 46(3), 305-316.

Walker, M., \& Thomson, P. (2010). The routledge doctoral supervisor's companion: Supporting effective in education and the social sciences. New York : Routledge.

Welsh, E. (2002). Dealing with data : Using Nvivo in the qualitative data analysis process. FQS, 3(2).

Wisker, G. (2005). The good supervisor : supervising postgraduate and undergraduate research for doctoral theses and dissertations. Basingstoke : Palgrave Macmillan.

Wright, T., \& Cochrane, R. (2000). Factors influencing successful submission of PhD theses. Studies in Higher Education, 25(2), 181-195.

Zuber-Skerritt, O. (1994). Improvising the quality of postgraduate supervision through residential staff development programmes. In O. Zuber-Skerritt \& Y. Ryan (Eds.), Quality in postgraduate education (pp. 77-89). London : Kogan Page.

\section{NOTES}

1. La licence française, équivalente du Bachelor dans la processus de Bologne, est d'une durée de trois ans.

2. Le master français est d'une durée de deux ans. La première année de master se nomme le master 1 ou M1. L'orientation vers un master recherche et un master professionnel a lieu à l'entrée en deuxième année de master (master 2 ou M2).

\section{RÉSUMÉS}

La supervision de recherche constitue une variable importante de la réussite en deuxième et troisième cycles universitaires. Pour réduire les échecs et les abandons à ces niveaux universitaires, bon nombre de recherches se focalisent sur le lien entre la qualité de la supervision et la réussite de l'étudiant. Or, la relation pédagogique de supervision est une relation duale, le superviseur n'est pas le seul acteur de la réussite de l'étudiant. Peu abordé dans la littérature, nous questionnons dans cet article le rôle de l'étudiant dans sa propre réussite. Des entretiens semi-directifs ont été menés auprès de superviseurs et d'étudiants, dans trois 
disciplines des sciences humaines et sociales et dans sept universités françaises. Les résultats de cette enquête montrent que l'étudiant est le principal acteur de sa réussite. Quelle que soit la qualité réelle ou perçue de sa supervision, l'étudiant en situation de réussite est celui qui parvient à faire preuve d'autodirection. Nos données montrent que l'autodirection se traduit de deux manières principales avec, d'une part la découverte des implicites et, d'autre part la capacité de compensations des « manques » réels ou perçus.

Research supervision is an important factor of success in master and doctoral studies. To reduce failure and attrition at this level, numerous studies have focused on the link between the quality of supervision and student success. Since supervision is a two-way relationship, the supervisor is not the sole actor in determining the student's success. In this article we investigate the littleresearched subject of the role of the student in his or her own success. Semi-directive interviews were conducted with supervisors and students in three disciplines in the social sciences and humanities in seven French universities. The results of this study show the student as the key actor in his or her success. Successful students were those who demonstrated self-direction, regardless of the real or perceived quality of supervision. Our data show that the self-directed student has two main characteristics, one being discoverying the implicit and the other being the ability to compensate real or perceived deficiencies.

\section{INDEX}

Mots-clés : autodirection, direction de mémoire, mémoire, relation pédagogique, supervision

Keywords : dissertation, pedagogical relationship, self-direction, student success, supervision

\section{AUTEUR}

\section{LAETITIA GERARD}

PhD Collaboratrice scientifique au Centre de Soutien à l'Enseignement (CSE) de l'Université de Lausanne

Chercheure associée au Laboratoire Inter-universitaire en Sciences de l'Education et de la Communication (LISEC-Nancy)

Université Nancy 2, département des sciences de l'éducation, campus lettres et sciences humaines

23 boulevard Albert $1^{\text {er }}$

54000 Nancy

Courriel : gerard.laetitia@gmail.com

Site web : http://www.laetitia-gerard.com/ 